\title{
Early Detection of Breast Cancer a Need in LMICs
}

\section{Salma Amin Rattani*}

Assistant Professor, School of Nursing and Midwifery, Aga Khan University Karachi, Pakistan

*Corresponding Author: Salma Amin Rattani, Assistant Professor, School of Nursing and Midwifery, Aga Khan University Karachi, Pakistan.
Received: February 10, 2020

Published: April 09, 2020

(C) All rights are reserved by Salma Amin

Rattani.

\begin{abstract}
Breast cancer is the most common cancer in women both in Low-and-Middle-Income Countries (LMICs) [1] like Nigeria, Pakistan [2] and India [3] and in High-Income Countries (HICs) [1]. These include Belgium, Denmark, France, the Netherlands, the United Kingdom, the United States, Australia, Germany, Italy, Australia, New Zealand, Switzerland, and Sweden [4]. However, breast cancerrelated mortality is high in LMICs [5,6] where the majority of the cases are diagnosed at the late stages of the disease [7]. Factors contributing to late diagnosis include lack of awareness about breast cancer, poverty, cultural and religious beliefs, misconceptions about the disease, fear of mastectomy, women's lack of autonomy in health-related decision making [6], lack of adequate diagnosis, and treatment facilities [8]. Consequently, when diagnosed the disease is at the $3^{\text {rd }}$ or $4^{\text {th }}$ stage of cancer. Whereas, more than $90 \%$ of women are diagnosed early when they have a locoregional disease in HICs [6]. The early diagnosis of cancer, combined with an accessible, and affordable effective treatment, results in improvements in both the stage of cancer at presentation and mortality from cancer [9].

Keywords: Breast Cancer; Low-and-Middle-Income Countries (LMICs); High-Income Countries (HICs)
\end{abstract}

\section{Introduction}

Breast cancer is estimated that in 2020,1.7million new cases of breast cancer present in LMICs and the huge discrepancy in survival chances continue with most of the breast cancer deaths (70\%) occurring in LMICs. Thus, breast cancer has tremendous public health significance and because primary prevention is still not available, efforts to promote early detection should be highlighted [8] and link to diagnosis and treatment without a delay. When done promptly, cancer may be detected at a potentially curable stage, improving survival and quality of life [9].

\section{Purpose of the Study}

This review aims to highlight the importance of early detection of breast cancer which is the most common cancer in women worldwide [10].

\section{Breast cancer detection}

The World Health Organization (WHO) in its guide to cancer early diagnosis describes three steps of early diagnosis. Step-1 is awareness and accessing care. Step- 2 is clinical evaluation, diagnosis, and staging. Step-3 is access to treatment [9].

Step-1: Awareness and accessing care

Women must be aware of breast cancer [11], specific symptoms of breast cancer, understand the urgency of these symptoms, over- come fear or stigma associated with cancer and be able to access primary care [9]. For raising awareness about breast cancer, Breast Self-Examination (BSE) is suggested to be used as a modality. Steps for BSE that Budakoglu., et al. (2007) tabled in their research are (1) Get in front of the mirror, (2) Have the upper body completely naked, (3) Put the hands on the waist, (4) Inspect whether there is any deformity of the breasts, (5) Lift the arms up, (6) Bend forward from the waist, (7) Inspect whether there is any retraction or deformity of the breasts, (8) Put a pillow or towel under the right/left shoulder while examining the right/left breast during examination in the supine position, (9) Put the right/left hand behind the head while examining the right/left breast, (10) Carry out the examination with the hand parallel to the breast, (11) Carry out vertical/ circular/segmental examination using 3 or 4 fingers, (12) Carry out the examination without taking the fingers off the breast, (13) Carry out the examination with the finger pads, (14) Examine all the breast area, (15) Check for any deformity of the nipple, (16) Compress the nipple and check for discharge, (17) Exam the auxiliary area [12]. It is described to be the least expensive early detection screening method and it does not require any advanced technology and physician intervention. Although, in randomized control trials on BSE has not shown improvement in mortality related to breast cancer. Nevertheless, BSE has been highlighted important for breast cancer awareness campaigns [8]. 
Awareness-raising campaigns are not just to be limited to the women but these are to be expanded to the families, and social networks. This need is supported by Kohler., et al. (2017) who explored the factors influencing delay in diagnosing breast cancer in women in Malawi, an LMIC. Participants in their research were 20 women with breast cancer. The data collection was through in-depth interviews. One of the findings indicates that, women sought help from their social networks. These women often told their husbands first, but they also disclosed their symptoms to a family member, neighbor, or friend, or they consulted village elders. Yet they missed the opportunity for early diagnosis. Hence, public awareness and clearing their misconceptions are also critically important for a comprehensive approach to improve the early detection of breast cancer [11]. Talib., et al. (2019) based on their study in Pakistan and Kenya, both are LMICs, assert that, for starters, an effective and feasible screening strategy should focus on identifying women with breast masses, and then ensuring accessible and affordable diagnostic and treatment pathways [13]. Health promotion efforts should be a priority and should focus on educating women on the signs and symptoms of breast cancer and encouraging them to perform regular breast self-exams as a means of self-awareness [13]. Rivera-Franco., et al. (2018) recommended that the emphasis is urgently needed on health education to promote early diagnosis of breast cancer and to highlight the importance of creating more public facilities that provide treatment, which are the key components for improvement of breast cancer care in LMICs [8]. Thus, in these countries, the public-private collaboration can potentially be extended to the training health professionals in primary care for delivering community-based education sessions and integrating breast cancer screening into existing women's health services $[13,14]$.

\section{Step-2: Clinical evaluation, diagnosis, and staging}

This step can be classified into three components: accurate clinical diagnosis, diagnostic testing and staging, and referral for treatment. This interval begins with an evaluation by the health-care provider [9]. Clinical evaluation includes Clinical Breast Examination (CBE) which successfully reduces stage at diagnosis, which may translate to improve breast cancer survival with longer followup. In addition, CBE may have an educational component whereby women with new breast lumps may seek care earlier [15]. Hence, there is a need to designing and implementing effective health educational programs that increase women's awareness about breast cancer and promote screening steps to reduce the burden affected by breast cancer among women in LMICs [13]. Training front-line health professionals in CBE as a screening method for breast cancer and appropriate referral linkages have the potential to increase the detection of breast cancer at an early stage in LMCs $[13,14]$. Talib., et al. (2019) describe Pakistan as an LMIC where they involved Lady Health Workers (LHWs); members in profes- sional cadre in the country and through LHWs women were taught for Breast Self-Examination (BSE) Women with an abnormal BSE were then referred to the primary health to undergo a CBE by a physician and women with abnormal CBEs were then referred for diagnostic mammography [13]. Although recent controversies arose regarding screening mammography in HICs, it is still widely practiced in HICs. In LMICs, many countries rely on mammography for early detection and screening [16]. Rivera-Franco., et al. (2018) published a randomized control report that mammography is the only single modality for the screening of breast cancer and it has demonstrated an improvement in breast cancer mortality [8]. Talib., et al. (2019) supported that the primary care providers should be well-trained in clinical breast exams and those with concerning breast masses should be referred for diagnostic mammograms and further work-up as needed. This approach, which prioritizes population-wide education and self and clinical breast exams are a cost-conscious interventions appropriate for LMICs and may well result in a population-wide shift toward early detection and better survival rates from breast cancer [13]. However, Talib., et al. (2019) also recommended that concerted research efforts to address the global burden of breast cancer will need to focus on bridging the knowledge gap between screening strategies that work in HIC versus those that can be effectively applied in LMICs [13].

An adequate history and physical examination, basic imaging, tissue diagnosis with Fine-Needle Aspiration (FNA), and/or biopsy according to availability of services and pathologists are required for diagnosis [16]. Staging should be performed to assess the extent of disease. Basic staging traditionally includes routine lab tests including Complete Blood Counts (CBC), Chest X-Ray (CXR) and abdominal ultrasound. In HICs Computed Tomography (CT) scans and nuclear bone scans, or even Positron Emission Tomography (PET) scans are used increasingly. In many LMICs, these may not be available. On the contrary, if these are accessible these should be regulated and used judiciously [16]. Hence, the accessibility to accurate clinical assessment, clinical diagnosis, diagnostic testing, pathology, cancer staging and appropriate coordination between services and patients, and their follow-ups are important in the prevention of delayed diagnosis [9].

\section{Step-3: Access to treatment}

The WHO states that, promoting early identification of cancer in the absence of appropriate access to treatment is not only ineffective but is also unethical. A significant percentage of patients who receive a cancer diagnosis do not initiate or complete treatment due to various barriers. These may be related to financial constraints, geographic location of the healthcare settings, unavailability or unaffordability of the transportation and treatment modalities, time, poverty and anxiety, and fear about cancer treatment [9]. Basic cancer treatment consists of one or a combination of treatment mo- 
dalities including surgery, radiation therapy, and systemic therapy, chemotherapy, and endocrine therapy $[9,16]$. Surgeries to remove breast cancer could be breast-conserving surgery or mastectomy. In breast-conserving surgery, the parts of breast containing cancer as well as some surrounding normal tissues are removed. How much breast is removed depends on where and how big the tumor. Mastectomy is a surgery in which the entire breast is removed, including al the breast tissue and sometimes other nearby tissues. Chemotherapy can be given before or after surgery. Chemotherapy drugs used for breast cancer include Anthracyclines, such as doxorubicin, Taxanes, such as paclitaxel (Taxol) and docetaxel (Taxotere), 5-fluorouracil (5-FU) or capecitabine, Cyclophosphamide (Cytoxan), and Carboplatin (Paraplatin). Most often, combinations of 2 or 3 of these drugs are used [17]. However, in many LMICs, basic treatment services are unavailable [9]. The provisions of these treatment modalities are important in improving access to treatment of breast cancer.

\section{Conclusion}

Breast cancer is the most common cancer in women globally. However, in LMICs, it is diagnosed at a late stage and its related mortality is high in these countries. It is important that the disease should be controlled through early diagnosis. This requires raising public awareness about breast cancer, teaching women about breast self-examination, improving access to clinical breast examination, and diagnosis, staging, and treatment. Promoting early identification of cancer in the absence of appropriate access to treatment is not only ineffective but is also unethical [9]. Hence, it is important that breast cancer treatment should be provided.

\section{Bibliography}

1. World Health Organization. "Global health estimates". World Health Organization (2013).

2. Ghoncheh Mahshid., et al. "Incidence and mortality and epidemiology of breast cancer in the world". Asian Pacific Journal of Cancer Prevention 17.S3 (2016): 43-46.

3. Malvia Shreshtha., et al. "Epidemiology of breast cancer in Indian women". Asia-Pacific Journal of Clinical Oncology 13.4 (2017): 289-295.

4. Unger-Saldaña Karla. "Challenges to the early diagnosis and treatment of breast cancer in developing countries". World Journal of Clinical Oncology 5.3 (2014): 465.

5. Martei Yehoda M., et al. "Breast cancer in low-and middle-income countries: Why we need pathology capability to solve this challenge". Clinics in Laboratory Medicine 38.1 (2018): 161-173.

6. Gutnik Lily A., et al. "Breast cancer screening in low-and middle-income countries: a perspective from Malawi". Journal of Global Oncology 2.1 (2016): 4.
7. World Health Organization. "Cancer. Breast cancer: prevention and control”. World Health Organization (2020).

8. Rivera-Franco., et al. "Delays in breast cancer detection and treatment in developing countries". Breast Cancer: Basic and Clinical Research 12 (2018): 1178223417752677.

9. World Health Organization. "Guide to cancer early diagnosis" (2017).

10. Zhang Ping., et al. "Exosome-mediated delivery of MALAT1 induces cell proliferation in breast cancer". OncoTargets and Therapy 11 (2018): 291.

11. Kohler Racquel E., et al. "A framework for improving early detection of breast cancer in sub-Saharan Africa: A qualitative study of help-seeking behaviors among Malawian women". Patient Education and Counseling 100.1 (2017): 167-173.

12. Budakoglu I Irem., et al. "The effectiveness of training for breast cancer and breast self-examination in women aged 40 and over". Journal of Cancer Education 22.2 (2007): 108.

13. Talib Zohray., et al. "Promoting Breast Cancer Awareness and Clinical Breast Examination in the LMIC: Experiences from Tajikistan, Pakistan and Kenya". Current Breast Cancer Reports 11.3 (2019): 152-157.

14. Wu Tsu-Yin and Joohyun Lee. "Promoting breast cancer awareness and screening practices for early detection in lowresource settings". European Journal of Breast Health 15.1 (2019): 18.

15. Yip CH., et al. "Early diagnosis of breast cancer in the absence of population-based mammographic screening in Asia". Current Breast Cancer Reports 10.3 (2018): 148-156.

16. El Saghir Nagi S., et al. "Breast Cancer Diagnosis and Treatment in Low-and Mid-Resource Settings: the Role of ResourceStratified Clinical Practice Guidelines". Current Breast Cancer Reports 10.3 (2018): 187-195.

17. Treating Breast Cancer. "Chemotherapy for Breast Cancer" (2020).

\section{Assets from publication with us}

- Prompt Acknowledgement after receiving the article

- Thorough Double blinded peer review

- Rapid Publication

- Issue of Publication Certificate

- High visibility of your Published work

Website: https://www.actascientific.com/

Submit Article: https://www.actascientific.com/submission.php Email us: editor@actascientific.com

Contact us: +919182824667 